\title{
El duelo en medicina desde la visión y piel del profesional sanitario
}

\section{Grief in medicine from the vision and skin of the healthcare professional}

\author{
Erik Gabriel Díaz Avila ${ }^{1}$, Marcela Fernández-Carrera Muchova ${ }^{2}$, Teresa Sánchez \\ Villanueva ${ }^{3}$, Vega Sánchez Calvo ${ }^{4}$ y Jesús Francisco Mesonero Robles ${ }^{5}$ \\ Estudiantes del Grado en Medicina de la Universidad de Salamanca;
}

, erik_diaz_1992@usal.es; https:/ / orcid.org/0000-0002-2590-9734; ${ }^{2}$, marcelafernandezmuchova@gmail.com; https://orcid.org/0000-0002-8188-8990; ${ }^{3}$, teresasvil@gmail.com; https://orcid.org/0000-0001-8505-5534; ${ }^{4}$, vegasc02@usal.es; https:/ / orcid.org/0000-0003-4119-764X; ; ${ }^{5}$ jesusmeso7@gmail.com; https:// orcid.org/0000-0001-8644-4881

Recibido: 3-3-21; Aceptado: 19-4-21; Publicado: 26-4-21

Resumen: El duelo es un proceso natural que han de pasar las personas tras la pérdida de un ser querido. Durante la actual pandemia COVID-19 los profesionales sanitarios han sido víctimas, pues se han tenido que enfrentar a una situación sin precedentes en la que se ha puesto en juego su propia salud mental. El objetivo principal del estudio ha sido realizar una revisión de la literatura, no sistemática, en las diferentes bases de datos científicas para analizar el duelo en el ámbito de la medicina ligado al duelo vivido por los profesionales sanitarios, centrado en la situación actual de crisis sanitaria, así como los problemas que añadió la pandemia COVID-19. La pandemia COVID-19 ha puesto de manifiesto la importancia del duelo y la despedida justa, la cual no se pudo dar en la mayoría de los casos. Es imperiosa la preparación que han de recibir los profesionales sanitarios para enfrentar dicho proceso, inlcuídos los futuros profesionales sanitarios. Queda de manifiesto la importancia de la capacitación y cuidado de la salud mental, tanto en pacientes, familiares, personal sanitario y futuros profesionales de la salud. Igualmente, es importante garantizar la resiliencia enfocada a los aspectos relacionados con el duelo.

Palabras clave: duelo, profesional sanitario, COVID-19, salud mental

Abstract: Grief is a natural process that people go through after the loss of a loved one. During the current COVID-19 pandemic, health professionals have been victims, as they have had to face an unprecedented situation in which their own mental health has been put at stake. The main objective of the study was to carry out a non-systematic review of the literature in the different scientific databases to analyze grief in the field of medicine linked to grief experienced by health professionals, focused on the current crisis situation health, as well as the problems added by the COVID-19 pandemic. The COVID-19 pandemic has highlighted the importance of mourning and a fair farewell, which could not be given in most cases. The preparation that health professionals must receive to face this process, including future health professionals, is imperative. The importance of training and mental health care is evident, both in patients, relatives, health personnel and future health professionals. Likewise, it is important to guarantee resilience focused on aspects related to grief.

Keywords: grief, professional health worker, COVID-19, mental health 


\section{Introducción}

La palabra duelo proviene de la raíz dōlu, término latino que se refiere al dolor o sufrimiento tras la pérdida de un ser querido. No debe confundirse con duelo patológico, ya que este último aparece al intensificarse las fases normales del duelo y, se acompaña de trastornos depresivos mayores. En la Clasificación Internacional de Enfermedades, $11^{\mathrm{a}}$ revisión (CIE-11) se recoge con el código QE62 al denominado duelo sin complicaciones, incluido junto a los problemas asociados con la ausencia, la pérdida o la muerte de otra persona. Por otra parte, y con el código 6B42, tenemos el trastorno por duelo prolongado o patológico que pertenece a la familia de trastornos relacionados con el estrés. A su vez, en el DSM-V, Manual Diagnóstico y Estadístico de los Trastornos Mentales desarrollado por la Asociación Estadounidense de Psiquiatría, se recoge con el código V62.82 (Z63.4) al duelo no complicado, siendo aquel que transcurre de manera normal tras la pérdida de un ser querido, aunque, pueden identificarse síntomas similares a los que acompañan a una depresión mayor, tal como el insomnio o la anorexia (1-3).

El duelo en medicina se identifica con las experiencias dolorosas y diferentes fases que sufre una persona tras la pérdida de un ser querido, aunque en algunos casos, dichas sensaciones pueden adelantarse a la muerte del ser si existe una preparación psíquica previa a que acontezca la muerte, por ejemplo, cuando ocurre la muerte a causa de la vejez o enfermedades crónicas. En este sentido, el duelo se percibe como un período de intenso contraste emocional, donde se alterna dolor, depresión, ansiedad o incluso, puede desencadenar trastornos del comportamiento y emocionales, principalmente si la muerte se debe a un proceso traumático y agudo, sin existir forma de asimilar los hechos. Las situaciones más influyentes que generan un duelo anómalo y profundo se deben al fallecimiento de niños y jóvenes $\mathrm{y}$, a su vez, los niños que han perdido a sus padres serán más vulnerables a sufrir trastornos psiquiátricos (4).

En estos momentos, el Sistema Nacional de Salud (SNS) español está sumido en una crisis sanitaria causada por el virus SARS-CoV-2, zoonosis registrada con inicio en Wuhan, China, en el mes de diciembre del año 2019 y que, ha dado lugar a la pandemia COVID-19. Actualmente, en el mes de abril de 2021, en España se registran más de 3,4 millones de casos confirmados y de estos cerca del $5 \%$ corresponde a personal sanitario, es decir, unos 80.000 profesionales del área de la salud. En cuanto a las cifras de fallecidos con causa SARS-Cov-2 establecida, en el mundo se registran cerca de 3 millones de personas, de las cuales más de 75.000 pertenecen a España (5).

\section{Métodos}

El presente artículo es una revisión de la literatura, no sistemática, centrado en el duelo y relacionado con la situación actual de COVID-19. En este sentido, se realizó una búsqueda en distintas bases de datos y páginas webs institucionales, tales como Pubmed, Science direct España y la web institucional de la Organización Mundial de la Salud (OMS), principalmente. Las palabras claves que se emplearon en las búsquedas en español han sido: duelo, profesional sanitario, COVID-19 y salud mental. Por otra parte, se realizó una búsqueda en inglés donde se usaron como palabras clave grief, sanitary professional, COVID-19, mental health. A su vez, se aplicaron como términos MESH en Pubmed grief y medicine junto al operador booleano AND, con el fin de recuperar aquellos artículos enfocados en el duelo y en el ámbito médico. Se incluyeron los artículos comprendidos en el período del año 2015 al 2020, ambos inclusive, y que contasen con texto completo libre y acceso gratuito en el momento de la revisión y búsqueda. 
Por otra parte, tras la búsqueda no sistemática en las páginas webs o en la extensión de Google (Google Académico) se incluyeron algunos trabajos que, a pesar de no estar comprendidos en el período fijado inicialmente, añadieron por su contenido información relevante al objetivo del estudio planteado y que ayudó a contrastar la información recabada. Por último, se han excluido y no han sido objeto del estudio, aquellos cuya temática principal no era el análisis del duelo en medicina o los artículos que no incidían en la relación duelo versus profesional sanitario, así como, los que, al abordar la situación pandémica, no la contrastaban con el duelo.

\section{Resultados y Discusión}

Dentro de la faceta clínica destaca el papel del médico, pues es quien porta el peso de dar la mala noticia a los seres queridos del paciente, por desgracia, esta situación se ha vivido de manera continua durante los últimos meses debido a la pandemia COVID-19. Tras la pérdida de una persona cercana, llega a desencadenarse estrés o incluso un trauma psíquico, pudiéndose observar a través de síntomas como sensación de frialdad, amargura o inseguridad. La situación actual ha provocado que muchas personas no se hayan despedido de sus seres queridos, lo que puede desencadenar en un futuro en el llamado duelo patológico. Además, hoy en día, el duelo es más difícil de llevar ya que se ha añadido el confinamiento y con él, las medidas que no han permitido a las personas despedirse de sus seres queridos. Es entonces cuando nos damos cuenta de la importancia que tienen las ceremonias o ritos de despedida, pues nos permiten estar rodeados de la gente de confianza y que más nos importa. Por tanto, una de las soluciones que se añade a la causa de la despedida justa, se fundamentó en las nuevas tecnologías, las cuales nos han facilitado la interacción con familiares o seres queridos ya que las visitas durante su estancia hospitalaria no eran posibles debido a la situación epidemiológica (6).

Cortés (2020) entrevistó a Ana María Egido Mendoza, psicóloga especialista en duelo, quien señala que "Es muy recomendable hablar con otras personas que han pasado por un duelo difícil antes que nosotros ya que, si ellas han podido soportarlo y seguir adelante nosotros también podremos hacerlo". El duelo puede tornarse difícil para cualquier ser humano, pero hay algunas circunstancias donde adquiere un mayor peso, por ejemplo, la muerte repentina de una persona ya sea por un accidente o por otras situaciones traumáticas como un suicidio o asesinato o puede darse el caso del fallecimiento de una persona joven o de alguien que gozaba de una inmensa salud. En contraposición se encuentra el fallecimiento de una persona de edad avanzada que necesite cuidados paliativos y tenga una muerte senil. A su vez, hay que tener en cuenta la relaciones personales existentes, porque dependiendo del grado afectivo o cercanía, se verán afectadas en mayor o en menor medida las personas que les corresponda vivir el duelo (7).

\section{Duelo y Dolor.}

La International Association for the Study of Pain (IASP), define el dolor como una experiencia sensorial o emocional desagradable. Por otra parte, la existencia de dolor no implica duelo, pero, el duelo suele estar acompañado de dolor y es en esta faceta donde se hace necesario que el profesional sanitario (PS) tenga unas buenas bases de conocimiento de los procesos y fases del duelo. A su vez, la muerte en soledad no solo causa sufrimiento a los que mueren, sino que puede dejar secuelas en forma de duelo patológico para los que estén cerca de los difuntos y también para los profesionales de la salud, quienes a veces son los únicos que proporcionan apoyo al paciente terminal y a su familia (8). A su vez, parte del trabajo del PS conlleva establecer vínculos con el difunto, adaptarse a las nuevas circunstancias de la vida y construir nuevas relaciones, de tal manera que el dolor es visto 
como un modo de orientación de la pérdida, centrado en las emociones, restauración y centrado en el afrontamiento del problema.

Por otra parte, el duelo anticipatorio es la preparación antes de ocurrir la pérdida, situación que disminuye la manifestación de dolor tras la partida del ser querido, característica que suele estar integrada en el PS y que le otorga mayor conciencia de la mortalidad. De este modo, la preparación psicológica es fundamental para enfrentar el duelo, pues es de gran ayuda adoptar una actitud serena, empática, mantener una escucha activa y principalmente, servir de apoyo emocional. El propio duelo y la forma en que cada uno lo vive está condicionado por factores como la edad, el género, la cultura y la religión, lo que a su vez hace complicado dar una respuesta o solución universal. Por lo tanto, los servicios psicológicos y psiquiátricos cumplen un rol fundamental para el acompañamiento en el duelo o superación de dolor, tanto en pacientes y familias como en otros compañeros del sector de la salud (9).

El duelo sin despedida, que ha prevalecido durante la actual pandemia COVID-19, acarrea trastornos psicosomáticos mayores en aquellas personas que lo han sufrido. Las manifestaciones derivadas de la pérdida de un ser querido suelen ser psicológicas y emocionales, ya que las familias han de exponerse a situaciones no agradables. En algunos casos, las personas afectadas tras el duelo llegan a manifestar los mismos síntomas que presentó el ser querido antes de morir, por lo tanto, son hechos que el PS ha de tener presente. A su vez, el PS sufre una alta carga mental y desgaste emocional porque, en muchos casos, la muerte no es el resultado que se espera y es en estas situaciones donde el PS no ha de gozar sólo de una salud física, sino también de salud e higiene mental. Por otra parte, se recomienda que el PS reciba formación específica de cómo asistir adecuadamente a pacientes terminales y a su vez, en cómo se pueden afrontar las distintas situaciones con las que ha de lidiar cara a los familiares de los pacientes y también a otros PS (10).

\section{COVID-19 vs Duelo.}

Tal vez, uno de los mayores impactos emocionales que podamos vivir, sea no poder estar junto a un ser querido en el momento de su partida. La pandemia COVID-19, hizo y aún hace enfrentarse al PS al duelo de manera continuada cómo antes nunca lo habían imaginado y, en este caso hablamos del duelo directo, el que se debate entre la vida y la muerte. En esta escena, podemos destacar la imposibilidad del contacto físico con los seres queridos en sus últimos momentos. Como ya se ha comentado, la situación de pandemia no posibilitó ese contacto, el que permite estrechar la mano fuertemente y mirar por última vez a los ojos de aquel que está muriendo, observando que van encontrando la muerte en paz, tranquilos, asumiendo el final de su vida, como sería esperado en el ciclo natural de la vida. Sin duda alguna, la pandemia COVID-19 provocó cambios sociales y sanitarios bruscos e impredecibles. Tal como detalla Unamuno en su obra "Niebla» “...entró de puntillas y sin ruido, como un ave peregrina, y se la llevó a vuelo lento, en una tarde de otoño. Murió con su mano en la mano de su hijo, con sus ojos en los ojos de él..." $(7,11)$.

En las distintas escuelas médicas del mundo, los médicos en formación y los profesionales aprenden las pautas que deben llevar a cabo en situaciones de duelo. Las respuestas de duelo atípicas pueden prolongar el sufrimiento, interrumpir las actividades normales y provocar un aumento de la mortalidad. Asimismo, un buen trabajo realizado por el PS contribuye a una mejoría en la lidia de los sentimientos, el sufrimiento e incluso, la aceptación de la muerte (7). 
Por una parte, el distanciamiento social es clave para frenar el contagio, pero aumentó la complejidad de la atención, el aislamiento y la ansiedad. La población mundial, se encuentra profundamente afectada por esta situación de incertidumbre, pero sobre todo (aunque en numerosas ocasiones no se haya tenido en cuenta), esto incidió directamente en el confort del PS. De este modo, el bienestar, incorpora la salud mental, física y espiritual, dedicada a luchar contra el agotamiento para ejercer de la mejor manera posible, esta profesión tan vocacional. A su vez, la falta del bienestar en los médicos y demás PS en la actual situación COVID-19, afectó directamente a su forma de enfrentarse a la enfermedad y brindar los cuidados necesarios (12).

\section{Duelo y Personal Sanitario.}

Es necesaria la comprensión de los factores estresantes que la crisis sanitaria producto del COVID-19 ejerció sobre los médicos, pues nos ayuda a reconocer lo que se necesita para restaurar la salud en su totalidad. Los médicos aprovecharon las herramientas que se les ofrecieron para recobrar la fuerza y el gran significado de su trabajo. A pesar de esto, les faltó la preparación para reconocer las señales de alerta que les ofrece su organismo para identificar los primeros signos de ansiedad, depresión, trastornos de estrés postraumático, entre otros y así, ser atendidos y poder evitar a tiempo situaciones agudas graves, tal como un desequilibrio emocional o psicológico -burnout o desgaste por compasión-. En este sentido, no todo el PS tiene en cuenta que si están mermadas sus capacidades cognitivas, no serán tan eficaces en su encomiable labor, en la que tuvieron que atender a pacientes en situaciones difíciles y tomar decisiones rápidas, hecho que no tuvo la mejor gestión posible, al menos al principio de la crisis, por la falta de efectivos de salud y de los medios de protección necesarios $(4,12)$.

En honor a los médicos fallecidos a consecuencia del SARS-COV-2 durante la pandemia COVID-19, se emitió el documental: "Vocación, el documental en honor a los médicos fallecidos por la COVID-19". Desde el inicio de la crisis sanitaria y hasta el momento presente, los médicos y PS no han dejado de prestar sus servicios o atender las consultas médicas a pesar de las distintas dificultades, todo ello sin perder la oportunidad de atender, diagnosticar, ayudar en la medida de lo posible, es decir, realizar su labor. Paralelamente a esto, en sus casas, decidieron tomar una serie de medidas, que se convertirían en la forma de expresar su protección y cariño a sus familiares, por ejemplo, dormir en el sofá, cenar a horas distintas, no ir a sus casas, entre otras medidas que garantizasen la seguridad de su hogar y que le permitirían seguir ejerciendo su vocación, medidas que a muchos les pueden parecer excesivas. A su vez, han realizado dobles e incluso triples jornadas, sin dormir, sopesando esa gran incertidumbre de la que antes hablábamos y, sobre todo, conscientes de que se exponían al contagio del virus (13).

En el documental "Vocación, el documental en honor a los médicos fallecidos por la COVID-19", sus familias narraron sus experiencias como médicos, pues les detallaron atentos y complacientes, ya fuese a su vecina, suegra, desconocido, atendiendo llamadas fuera del horario de trabajo, dedicando horas a la investigación, guardando expedientes, sin olvidarse en ningún momento de cada uno de ellos, etc. No hay que olvidar a los médicos y PS que fallecieron, estando a pie de cañón, haciendo lo que realmente amaban, quienes se dieron a los demás hasta el último momento, hasta el punto de decidir no utilizar un respirador para que lo pudiese utilizar otra persona (13).

Abordaje del duelo.

Existen medidas que pueden ayudar a sobrellevar el duelo, entre ellas, la intervención telepsicológica o los rituales de despedida alternativos, como escribir una carta o crear una 
caja de recuerdos. Entre marzo y mayo de 2020, el Ministerio de Salud y la Asociación Española de Psicología crearon el Servicio de Primera Ayuda Psicológica-SPAP, una línea de ayuda nacional diseñada para brindar una intervención psicológica temprana a las personas afectadas por COVID-19. Los resultados mostraron que fue mayormente utilizada por mujeres, en el rango de edad entre 40 y 59 años, siendo las preguntas más frecuentes aquellas sobre la ansiedad y síntomas depresivos (14-15).

\section{Problemas y Soluciones.}

Queda recordar que el duelo, no tiene lugar solo post mortem, sino que este puede empezar cuando se diagnostica la enfermedad terminal. En este caso, a partir de cuándo se informa el proceso patológico, la relación médico-paciente queda sujeta a la ansiedad y estados depresivos que puedan generarse en el enfermo y sus familiares e incluso, pueden afectar al médico anímicamente. A su vez, existen diversas actitudes del PS que pueden condicionar un empeoramiento de la situación, por ejemplo, no querer referirse a la muerte y de cierta forma, enmascarar la verdad al paciente sobre la gravedad de su estado de salud, lo que se traduce en una actitud paternalista. En otras ocasiones, se dan los casos en los que algunos sanitarios no miran cara a cara a los pacientes moribundos por temor. Lo grave es que el enfermo lo nota y se ve a sí mismo como un apestado o rechazado, lo que puede parecer que el PS está evitando pensar en la muerte y afrontarla. En otras situaciones, es debido a este sentimiento de culpa e impotencia, por lo que el PS intenta compensarlo con el uso de las nuevas tecnologías. Es decir, de cierta forma, se han empeorado las condiciones de muerte, convirtiéndose el morir hospitalario en algo "solitario, mecánico y deshumanizado" (16-17).

Para solucionar esta situación de aislamiento, es necesario una formación y unas actitudes adecuadas hacia el enfermo, por ejemplo, hacer conciencia de las necesidades del paciente, observar y escuchar para identificar sus preocupaciones e intentar ayudar en todo lo posible para aliviar su dolor físico y psíquico. A su vez, facilitar y disponer las mejores condiciones posibles del entorno, que hagan más cómodo y acogedor el habitáculo del enfermo. Por otra parte, es necesario atender adecuadamente las necesidades informativas tanto del paciente como de los familiares, sin ocultar información a menos que éste lo pida expresamente. Lo dispuesto anteriormente parte de la ley 41/2002, de 14 de noviembre, básica reguladora de la autonomía del paciente y de derechos y obligaciones en materia de información y documentación clínica, en cuyo artículo 4 se cita (17-18):

"Los pacientes tienen derecho a conocer, con motivo de cualquier actuación en el ámbito de su salud, toda la información disponible sobre la misma, salvando los supuestos exceptuados por la Ley. Además, toda persona tiene derecho a que se respete su voluntad de no ser informada. La información, que como regla general se proporcionará verbalmente dejando constancia en la historia clínica, comprende, como minimo, la finalidad y la naturaleza de cada intervención, sus riesgos y sus consecuencias" (18).

Con la situación actual COVID-19, un mayor número de personas han necesitado terapia para superar las pérdidas y enfrentar el duelo, estando afectados tanto el PS como pacientes y familiares. Para promover la salud integral del personal sanitario en la situación actual, se ha intentado promover la fortaleza psicológica, ya que por defecto el PS antepone el bienestar del paciente al suyo propio tal como ocultar sus inquietudes, preocupaciones y miedos. Por ejemplo, actualmente un sentimiento que se ha generalizado en el PS es el sentimiento de culpa ante la pérdida de un paciente, ante el contagio, etc. Algunas medidas llevadas a cabo han sido las relacionadas con la gestión de los estados 
emocionales del PS. Frente a esta situación se procede a aislar al sujeto del grupo y ayudarle a ver la situación con realismo (que no es culpable del contagio, que ha hecho lo que ha podido por el paciente, entre otros ejemplos), ayudar a entender que es una reacción común ante la pérdida/enfermedad, así como el fomento de equipos de apoyo al PS para que practiquen la escucha activa (19).

Una de las causas de un duelo mal gestionado, está íntimamente relacionada con la educación recibida y ligado a la propia cultura de las poblaciones, pues, normalmente se trata a la muerte como tema tabú, lo cual complica la aceptación y normalización de ésta. Lo antes mencionado, se debe en parte a los avances de la medicina, que han permitido mejorar el nivel de vida y ha reducido la mortalidad, traduciéndose en un incremento de la esperanza de vida, lo que dificulta que se acepte la muerte cuando ocurre en edades tempranas o con gran expectativa de vida. Una de las formas para facilitar el duelo y que se puede aplicar al PS como a los familiares, es crear una nueva visión de la muerte y su inevitabilidad, lo que se traduce en una mejor aceptación de esta (18).

Aparte de esto, se han creado cursos y formaciones postgrado para el PS con el fin de acercarles más a la situación de duelo y estrés, con lo que se consigue que se familiaricen con las situaciones de duelo, dolor y pérdida de los seres queridos y pacientes. Un ejemplo de formación postgrado es la de "Formación de residentes en depresión y duelo", cuyos objetivos fueron mejorar el conocimiento sobre el cuidado de la depresión, así como mejorar las actitudes y el nivel de comodidad al brindar atención al duelo y a la depresión (20).

Como alternativas de apoyo frente al duelo podemos destacar, además de las ayudas que reciben tanto PS como familiares por parte de otros profesionales, una plataforma digital realizada por el Canadian Virtual Hospice en colaboración con socios canadienses, que desarrollaron MyGrief.ca. Esta es una herramienta psicoeducativa online para los que no pueden acceder a apoyo presencial para superar, afrontar la pérdida del ser querido y el duelo, sirviendo a su vez como recurso complementario para los que sí reciben dicha ayuda. Actualmente, con la situación COVID-19, este tipo de ayudas son las que sin duda alguna deben promoverse y fomentarse, tanto en el ámbito sociosanitario como a los pacientes (21).

Por último, hay que destacar el Proyecto de Innovación Docente "La sensibilización precoz hacia la muerte en la adquisición de competencias actitudinales en estudiantes de Medicina", encontrándose en fase clínica en el momento de su consulta. En dicho Proyecto, se pretende valorar la adquisición de competencias de estudiantes de Medicina en relación con las capacidades que puedan adquirir para enfrentar la pérdida de los pacientes, la muerte, abordar las peores situaciones clínicas, en definitiva, competencias que han de preparar para afrontar el duelo y ayudar a afrontarlo a aquellos que lo sufren (22).

\section{Conclusiones}

- El duelo en medicina es una situación inevitable ya que es parte natural de la vida y en algún momento nos toca vivirlo, ya sea el duelo como individuo, como familiar o como profesional sanitario.

- Las principales afectaciones que provoca el duelo están directamente relacionadas con la salud mental, pues los cambios en la persona suelen ir desde la no aceptación de la defunción ligado al enfado, hasta una profunda depresión que se agrava con el duelo patológico que aparece cuando se perpetúa en el tiempo la no superación de la muerte. 
- La actual crisis sanitaria, producto de la pandemia COVID-19, ha puesto de manifiesto la importancia de la despedida y el contacto cercano entre los familiares y el ser querido, cuya vida se acaba. A su vez, la situación actual ha permitido evidenciar que los profesionales sanitarios también sufren el duelo, pero sería correcto poner en duda si existe la preparación adecuada y el suficiente apoyo psicológico hacia los profesionales sanitarios que han de luchar cara a cara y, de manera reiterada con la muerte de compañeros, familiares y pacientes.

- La salud mental goza cada vez más de una mayor importancia, principalmente en aquellos aspectos que se han analizado en el presente trabajo, existiendo diferentes líneas de estudio y de apuesta por garantizar la higiene y salud mental de los pacientes, familiares y personal sanitario.

- Queda por destacar la importancia que se le da a la resiliencia y capacitación de los estudiantes de Medicina ya que, la situación vivida debe servirnos de ejemplo para fortalecer la resiliencia y la salud mental del que será el futuro profesional sanitario.

Financiación: No ha habido financiación.

Agradecimientos: En primer lugar, agradecer a la Dra. Bertha M. Gutiérrez Rodilla, quien ha sido nuestra guía en la asignatura Bases Metodológicas del Conocimiento Científico y quien ha posibilitado nuestra inspiración para realizar dicho artículo académico, el cual está dedicado a todos los profesionales sanitarios y pacientes, así como a las víctimas de la pandemia COVID-19. A su vez, darle las gracias a familiares y amigos por siempre estar para apoyarnos.

Declaración de conflicto of interés: Los autores declaran no tener ningún conflicto de intereses.

\section{Referencias}

1. Diccionario de términos médicos. Duelo [Internet]. España: RANM; 2012 [citado 23 Nov 2020]. Disponible en: https://dtme.ranm.es/buscador.aspx?NIVEL_BUS=3\&LEMA_BUS=duelo

2. Clasificación Internacional de Enfermedades-11 [Internet]. Estadísticas de mortalidad y morbilidad. $11^{\mathrm{a}}$ [citado 23 Nov 2020]. Disponible en: https://icd.who.int/browse11/l-m/es

3. American Psychiatric Association [Internet]. Guía de consulta de los criterios diagnósticos del DSM-5TM. Estados Unidos, 2013. 451 p. Disponible en: https://www.eafit.edu.co/ninos/reddelaspreguntas/Documents/dsm-v-guia-consulta-manualdiagnostico-estadistico-trastornos-mentales.pdf

4. Soklaridis S, Ferguson G, Bonato S, Saikaly R, Mosher PJ. Being there: protocol for a scoping review of the medical education literature on grief support training for medical professionals. BMJ Open 2018;8: e022778. http://doi.org/10.1136/bmjopen-2018-022778

5. Instituto de Salud Carlos III (ISCIII) [Internet]. Informes COVID-19. Informe ${ }^{\circ} 4$. Situación de COVID-19 en España a 14/4/2021; 2021. Disponible en: https://www.isciii.es/QueHacemos/Servicios/VigilanciaSaludPublicaRENAVE/ EnfermedadesTransmisibles/Documents/INFORMES/Informes\%20COVID-19/INFORMES\%20COVID19\%202021/Informe\%20COVID-19.\%20N\%C2\%BA\%2074_14\%20de\%20abril\%20de\%202021.pdf

6. Cortés N. "El duelo por la Covid-19 es complejo y puede convertirse en patológico si no se trabaja en ello" [Internet]. ConSalud; 2020 [citado 29 Nov 2020]. Disponible en: https:/ / www.consalud.es/la-entrevista/duelo-covid-19-complejo-convertirse-patologicono $81530 \quad 102 . \mathrm{html}$

7. Cabodevilla I. Las pérdidas y sus duelos. Anales Sis San Navarra [Internet]. 2007 [citado 29 Nov 2020]; 30 (3): 163-176. Disponible en: http://scielo.isciii.es/scielo.php?script=sci_arttext\&pid=S113766272007000600012\&lng=es

8. Vieta E, Pérez V, Arango C. Psychiatry in the aftermath of COVID-19. Rev Psiquiatr Salud Ment. 2020; 13(2): 105-100. Disponible en: http://doi.org/10.1016/j.rpsm.2020.04.004

9. Hamilton IJ. Understanding grief and bereavement. Br J Gen Pract. 2016; 66(651): 523. Disponible en: http://doi.org/10.3399/bjgp16X687325 
10. Rosado Pérez G, Varas Díaz N. Experiencias y proceso de duelo entre profesionales de enfermería oncológica pediátrica. Rev Puertorriquena Psicol. 2020; 30(2): 290-306. Disponible en: https://www.ncbi.nlm.nih.gov/pmc/articles/PMC7195809/

11. Unamuno M. Niebla. Madrid: Renacimiento; 1914. P. 30

12. Areahumana.es [Internet]. Superar el duelo cuando el Covid-19 lo ha cambiado todo. 2020 [citado 30 Nov 2020]. Disponible en: https://www.areahumana.es/superar-el-duelo-coronavirus/

13. Ilustre Colegio Oficial de Médicos de Madrid. Vocación, el documental en honor a los médicos fallecidos por la COVID-19 [vídeo en internet]. Youtube. 16 de Julio de 2020. [citado 24 Nov 2020]. Disponible en: https://www.youtube.com/watch?v=NsYcj7CP 6s

14. Berdullas Saunders S, Gesteira Santos C, Morán Rodríguez N, Fernández Hermida JR, Santolaya F, Sanz Fernández J, et al. El teléfono de asistencia psicológica por la COVID-19 del Ministerio de Sanidad y del Consejo General de la Psicología de España: características y demanda. Rev Esp Salud Pública. 2020;94: [e113. Disponible en: https://www.mscbs.gob.es/biblioPublic/publicaciones/recursos_propios/ resp/revista cdrom/VOL94/C ESPECIALES/RS94C 202010138.pdf

15. Moriconi V, Valero E. El duelo durante la pandemia de COVID-19. Jul/Agos 2020 [citado 30 Nov 2020]. En Mente y Cerebro: 103. Investigación y Ciencia [Internet]. Disponible en: https:// www.investigacionyciencia.es/revistas/mente-y-cerebro/mi-verdadero-yo-804/el-duelo-durantela-\%20pandemia-de-covid-19-18792

16. Gómez Esteban R. El médico frente a la muerte. Rev Asoc Esp Neuropsiq. 2012; 32 (113): 67-82. Disponible en: http:/ / doi.org/10.4321/S0211-57352012000100006

17. Gala León FJ, Lupiani Jiménez M, Raja Hernández R, Guillén Gestoso C, González Infante JM, Villaverde Gutiérrez $\mathrm{M}^{\mathrm{a}} \mathrm{C}$, Alba Sánchez I. Actitudes psicológicas ante la muerte y el duelo: Una revisión conceptual. Cuad med forense. 2002; 30: 39-50. Disponible en: https://scielo.isciii.es/scielo.php? script $=$ sci arttext\&pid $=$ S1135-76062002000400004

18. Ley 41/2002, de 14 de noviembre, básica reguladora de la autonomía del paciente y de derechos y obligaciones en materia de información y documentación clínica. (Boletín Oficial del Estado, número 274, de 15 de noviembre de 2002). En https:/ / www.boe.es/buscar/act.php?id=BOE-A-2002-22188

19. Cruz Almaguer AY, Gutiérrez Alvarez AK, Zaldivar Santos ED. Gestión de seguridad psicológica del personal sanitario en situaciones de emergencia por COVID-19 en el contexto hospitalario o de aislamiento. Rev Cubana Enferm. 2020; 36(2): e3704. Disponible en: http://www.revenfermeria.sld.cu/index.php/enf/article/view/3704

20. Ghesquiere A, Martinez J, Jalali C, Sirey JA, Morales S. Training residents in depression and grief. Clin Teach. 2018; 15(2): 114-119. Disponible en: http:/ / doi.org/10.1111/tct.12636

21. Cory S. MyGrief.ca. Ann Palliat Med 2018;7(Suppl 1):AB017. http://doi.org/10.21037/apm.2018.s017

22. Rodríguez Sánchez JA. Proyecto de Innovación Docente. La sensibilización precoz hacia la muerte en la adquisición de competencias actitudinales en estudiantes de Medicina: fase clínica. Memoria provisional: ID2016/205 [Internet]. 2017 [citado 2 Dic 2020]. Disponible en: https://gredos.usal.es/bitstream/handle/10366/135519/MID 16 205.pdf?sequence=1\&isAllowed=y

(C) 2021 por los autores. Enviado para su publicación en acceso abierto bajo los términos y condiciones de la licencia Creative Commons Attribution (CC BY) (http://creativecommons.org/licenses/by/4.0/). 